\title{
Four-loop wave function renormalization in QCD and QED
}

\author{
Peter Marquard, ${ }^{1}$ Alexander V. Smirnov, ${ }^{2}$ Vladimir A. Smirnov, ${ }^{3}$ and Matthias Steinhauser ${ }^{4}$ \\ ${ }^{1}$ Deutsches Elektronen-Synchrotron, DESY, 15738 Zeuthen, Germany \\ ${ }^{2}$ Research Computing Center, Moscow State University, 119991 Moscow, Russia \\ ${ }^{3}$ Skobeltsyn Institute of Nuclear Physics of Moscow State University, 119991 Moscow, Russia \\ ${ }^{4}$ Institut für Theoretische Teilchenphysik, Karlsruhe Institute of Technology (KIT), \\ 76128 Karlsruhe, Germany
}

(Received 28 January 2018; published 29 March 2018)

\begin{abstract}
We compute the on-shell wave function renormalization constant to four-loop order in QCD and present numerical results for all coefficients of the $\mathrm{SU}\left(N_{c}\right)$ color factors. We extract the four-loop Heavy Quark Effective Theory anomalous dimension of the heavy-quark field and also discuss the application of our result to QED.
\end{abstract}

DOI: $10.1103 /$ PhysRevD.97.054032

\section{INTRODUCTION}

Heavy quarks play an important role in modern particle physics, in particular in the context of QCD. This concerns both virtual effects, the production of massive quarks at collider experiments, and the study of bound state effects of heavy quark-antiquark pairs.

Processes which involve heavy quarks require the renormalization constants for the heavy-quark mass and, when they appear as external particles, also for the quark wave function. The mass renormalization constant in the on-shell scheme, $Z_{m}^{\mathrm{OS}}$, has been computed to four-loop order in Refs. [1,2]. In this work, we compute the wave function renormalization constant in the on-shell scheme, $Z_{2}^{\mathrm{OS}}$, to the same order in perturbation theory. $Z_{2}^{\mathrm{OS}}$ is needed for all processes involving external heavy quarks to obtain properly normalized Green's functions as dictated by the Lehmann-Symanzik-Zimmermann reduction formula. Currently, there is no immediate application for the fourloop term of $Z_{2}^{\mathrm{OS}}$. However, it is an important building block for future applications. For example, it enters all processes which involve the massive four-loop form factor. $Z_{2}^{\mathrm{OS}}$ is also needed for the five-loop corrections to static properties like the anomalous magnetic moment of quarks or, in the case of QED, of leptons.

The calculation of $Z_{2}^{\mathrm{OS}}$ is for several reasons more involved than the one of $Z_{m}^{\mathrm{OS}}$. First of all, one has to compute the derivative of the fermion self-energy, which leads to higher powers of propagators and thus to a more

Published by the American Physical Society under the terms of the Creative Commons Attribution 4.0 International license. Further distribution of this work must maintain attribution to the author(s) and the published article's title, journal citation, and DOI. Funded by SCOAP. involved reduction problem. Furthermore, $Z_{2}^{\mathrm{OS}}$ contains both ultraviolet and infrared divergences. Thus, dividing $Z_{2}^{\mathrm{OS}}$ by its $\overline{\mathrm{MS}}$ counterpart does not lead to a finite quantity as in the case of $Z_{m}^{\mathrm{OS}}$. $Z_{2}^{\mathrm{OS}}$ also depends on the QCD gauge parameter, whereas $Z_{m}^{\mathrm{OS}}$ does not.

The on-shell renormalization constants $Z_{2}^{\mathrm{OS}}$ and $Z_{m}^{\mathrm{OS}}$ can be extracted from the quark propagator by demanding that the quark two-point function has a zero at the position of the on-shell mass and that the residue of the propagator is $-i$. In the following, we briefly sketch the derivation of the relations between the heavy-quark self-energy and $Z_{2}^{\mathrm{OS}}$ and $Z_{m}^{\mathrm{OS}}$.

The renormalized quark propagator is given by

$$
S_{F}(q)=\frac{-i Z_{2}^{\mathrm{OS}}}{q-m^{0}+\Sigma(q, M)},
$$

where the renormalization constants are defined as

$$
\begin{aligned}
& m^{0}=Z_{m}^{\mathrm{OS}} M, \\
& \psi^{0}=\sqrt{Z_{2}^{\mathrm{OS}}} \psi .
\end{aligned}
$$

$\psi$ is the quark field with mass $m, M$ is the on-shell mass, and bare quantities are denoted by a superscript $0 . \Sigma$ denotes the quark self-energy, which is conveniently decomposed as

$$
\Sigma(q, m)=m \Sigma_{1}\left(q^{2}, m\right)+(\not q-m) \Sigma_{2}\left(q^{2}, m\right) .
$$

In the limit $q^{2} \rightarrow M^{2}$, we require

$$
S_{F}(q) \stackrel{q^{2} \rightarrow M^{2}}{\longrightarrow} \frac{-i}{q-M} .
$$


The calculation outlined in Ref. [3] for the evaluation of $Z_{m}^{\mathrm{OS}}$ and $Z_{2}^{\mathrm{OS}}$ reduces all occurring Feynman diagrams to the evaluation of on-shell integrals at the bare mass scale. In particular, it avoids the introduction of explicit counterterm diagrams. We find it more convenient to follow the more direct approach described in Refs. [4,5], which requires the calculation of diagrams with mass counterterm insertion.

Following Refs. [3-6], we expand $\Sigma$ around $q^{2}=M^{2}$ and obtain

$$
\begin{aligned}
\Sigma(q, M) \approx & M \Sigma_{1}\left(M^{2}, M\right)+(\not 1-M) \Sigma_{2}\left(M^{2}, M\right) \\
& +\left.M \frac{\partial}{\partial q^{2}} \Sigma_{1}\left(q^{2}, M\right)\right|_{q^{2}=M^{2}}\left(q^{2}-M^{2}\right)+\cdots \\
\approx & M \Sigma_{1}\left(M^{2}, M\right)+(\not 1-M)\left(\left.2 M^{2} \frac{\partial}{\partial q^{2}} \Sigma_{1}\left(q^{2}, M\right)\right|_{q^{2}=M^{2}}+\Sigma_{2}\left(M^{2}, M\right)\right)+\cdots
\end{aligned}
$$

Inserting Eq. (5) into Eq. (1) and comparing to Eq. (4) leads to the following formulas for the renormalization constants:

$$
\begin{aligned}
Z_{m}^{\mathrm{OS}} & =1+\Sigma_{1}\left(M^{2}, M\right), \\
\left(Z_{2}^{\mathrm{OS}}\right)^{-1} & =1+\left.2 M^{2} \frac{\partial}{\partial q^{2}} \Sigma_{1}\left(q^{2}, M\right)\right|_{q^{2}=M^{2}}+\Sigma_{2}\left(M^{2}, M\right) .
\end{aligned}
$$

Thus, $Z_{m}^{\text {OS }}$ is obtained from $\Sigma_{1}$ for $q^{2}=M^{2}$. To calculate $Z_{2}^{\mathrm{OS}}$, one has to compute the first derivative of the selfenergy diagrams. The mass renormalization is taken into account iteratively by calculating lower-loop diagrams with zero-momentum insertions.

It is convenient to introduce $q=Q(1+t)$ with $Q^{2}=$ $M^{2}$ and rewrite the self-energy as

$$
\begin{aligned}
\Sigma(q, M)= & M \Sigma_{1}\left(q^{2}, M\right)+(\emptyset-M) \Sigma_{2}\left(q^{2}, M\right) \\
& +t \emptyset \Sigma_{2}\left(q^{2}, M\right) .
\end{aligned}
$$

Let us now consider the quantity $\operatorname{Tr}\left\{\frac{\phi+M}{4 M^{2}} \Sigma\right\}$ and expand it to first order in $t$, which leads to

$$
\begin{aligned}
\operatorname{Tr}\left\{\frac{\mathscr{Q}+M}{4 M^{2}} \Sigma(q, M)\right\} & =\Sigma_{1}\left(q^{2}, M\right)+t \Sigma_{2}\left(q^{2}, M\right) \\
& =\Sigma_{1}\left(M^{2}, M\right)+\left(\left.2 M^{2} \frac{\partial}{\partial q^{2}} \Sigma_{1}\left(q^{2}, M\right)\right|_{q^{2}=M^{2}}+\Sigma_{2}\left(M^{2}, M\right)\right) t+\mathcal{O}\left(t^{2}\right) .
\end{aligned}
$$

The comparison to Eq. (6) shows that the leading term provides $Z_{m}^{\mathrm{OS}}$ and the coefficient of the linear term in $t$ leads to $Z_{2}^{\mathrm{OS}}$.

In the next section, we present results for $Z_{2}^{\mathrm{OS}}$ up to four loops, and in Sec. III, we discuss consistency checks which are obtained from matching full QCD to Heavy Quark Effective Theory (HQET). Section IV contains a brief summary and our conclusions.

\section{RESULTS FOR $Z_{2}^{\mathrm{OS}}$}

The wave function renormalization constant is conveniently cast into the form

$$
Z_{2}^{\mathrm{OS}}=1+\sum_{j \geq 1}\left(\frac{\alpha_{s}^{0}(\mu)}{\pi}\right)^{j}\left(\frac{e^{\gamma_{E}}}{4 \pi}\right)^{-j \epsilon}\left(\frac{\mu^{2}}{M^{2}}\right)^{j \epsilon} \delta Z_{2}^{(j)},
$$

where the bare strong coupling constant $\alpha_{s}^{0}$ has been used for the parametrization. Note that $\delta Z_{2}^{(i)}$ for $i \geq 3$ depend on the bare QCD gauge parameter $\xi$, which is introduced in the gluon propagator via

$$
D_{g}^{\mu \nu}(q)=-i \frac{g^{\mu \nu}-\xi \frac{q^{\mu} q^{\nu}}{q^{2}}}{q^{2}+i \varepsilon}
$$

With these choices, we can define the coefficients $\delta Z_{2}^{(i)}$ such that they do not contain $\log \left(\mu^{2} / M^{2}\right)$ terms. In fact, they can be combined with the factors $\left(\mu^{2} / M^{2}\right)^{j e}$ where $j$ is the loop order [cf. Eq. (9)]. The renormalization of $\alpha_{s}$ and $(\xi-1)$ is multiplicative so that, if required, $\alpha_{s}^{0}$ and $\xi^{0}$ can be replaced in a straightforward way by their renormalized counterparts using the relations

$$
\begin{aligned}
& \alpha_{s}^{0}=\left(\mu^{2}\right)^{2 \epsilon} Z_{\alpha_{s}} \alpha_{s}, \\
& \xi^{0}-1=Z_{3}(\xi-1),
\end{aligned}
$$

where 


$$
\begin{aligned}
Z_{\alpha_{s}} & =1+\frac{1}{\epsilon}\left(\frac{n_{f}}{6}-\frac{11}{12} C_{A}\right) \frac{\alpha_{s}}{\pi}+\cdots \\
Z_{3} & =1+\frac{1}{\epsilon}\left[-\frac{n_{f}}{6}+\left(\frac{5}{12}+\frac{1}{8} \xi\right) C_{A}\right] \frac{\alpha_{s}}{\pi}+\cdots
\end{aligned}
$$

$C_{A}=3$ is a $\mathrm{SU}(3)$ color factor, and $n_{f}$ is the number of active quarks. The ellipses denote higher-order terms in $\alpha_{s}$. To obtain the ultraviolet-renormalized version of $Z_{2}^{\mathrm{OS}}$, we need $Z_{\alpha_{\mathrm{s}}}$ to three loops and $Z_{3}$ to one-loop order. Note that in Eq. (9) it is assumed that the heavyquark mass is renormalized on shell; i.e., all mass renormalization counterterms from lower-order diagrams are included.

For the calculation of the four-loop diagrams, we proceeded in the same way as for the calculation of the mass renormalization constant $[1,2]$ and the muon anomalous magnetic moment [7] and thus refer to Ref. [2] for more details. Let us still describe some complications. After a tensor reduction, we obtain Feynman integrals from the same hundred families with 14 indices as in Refs. [1,2]. The maximal number of positive indices is 11 . One can describe the complexity of integrals of a given sector (determined by a decomposition of the set of indices into subsets of positive and nonpositive indices) by the number $\sum\left|a_{i}-n_{i}\right|$, where the index $n_{i}=1$ or 0 characterizes a given sector. What is most crucial for the feasibility of an integration-by-parts (IBP) reduction is the complexity of input integrals in the top sector, i.e., with $n_{i}=1$ for $i=$ $1,2, \ldots, 11$ and $n_{i}=0$ for $i=12,13,14$. In the present calculation, this number was up to 6 , while in our previous calculation it was 5 . Therefore, the reduction procedure performed with FIRE [8-10] coupled with LITERED [11,12] and CRusher [13] was essentially more complicated as compared to that of Refs. [1,2].

As in Refs. [1,2], we revealed additional relations between master integrals of different families using symmetries and applied the code TSORT, which is part of the latest FIRE version [10]. In most cases, the master integrals were computed numerically with the help of FIESTA [14-16]. For some master integrals, we used analytic results obtained by a straightforward loop-by-loop integration at general dimension $d$ and also used analytical results obtained for the 13 nontrivial four-loop on-shell master integrals computed in Ref. [17]. As is described in detail in Ref. [2], we also applied Mellin-Barnes representations [18-21]. In the case of one-fold Mellin-Barnes representations, it is possible to obtain a very high precision (up to 1000 digits) so that analytic results can be recovered using the PSLQ algorithm [22]. Often the two-, three-, and higher-fold Mellin-Barnes representations provide a better precision than FIESTA. Recently, a subset of the master integrals has been calculated either analytically or with high numerical precision, in the context of the anomalous magnetic moment of the electron [23]. However, these results are not available to us.

The more complicated IBP reduction resulted in higher $\epsilon$ poles in the coefficients of some of the master integrals, so that the corresponding results are needed to higher powers in $\epsilon$. Depending on the integral, we either straightforwardly evaluated more terms with FIESTA or obtained more analytical terms or more numerical terms via MellinBarnes integrals.

Let us mention that we compute the self-energies on the right-hand side of Eq. (6) including terms of order $\xi^{2}$. We did not evaluate the $\xi^{3}, \xi^{4}$, and $\xi^{5}$ contributions. Some diagrams develop $\xi^{6}$ terms, which we reduced to master integrals, and we could show that their contributions to $Z_{2}^{\mathrm{OS}}$ add up to zero. Thus, our final result for $Z_{2}^{\mathrm{OS}}$ contains $\xi^{2}$ terms. We cannot exclude that also higher-order $\xi$ terms are present but we do not expect that there are $\xi^{n}$ terms present in $Z_{2}^{\mathrm{OS}}$ for $n \geq 4$.

Let us in a first step turn to the one-, two-, and three-loop results for $Z_{2}^{\mathrm{OS}}$, which are available from Refs. [5,6,24]. We have added higher-order $\epsilon$ terms, which are necessary to obtain $Z_{2}^{\mathrm{OS}}$ at four loops. In Appendix B, we present results which in particular include the $\mathcal{O}(\epsilon)$ terms of the three-loop coefficient.

In the following, we present results for all $23 \mathrm{SU}\left(N_{c}\right)$ color structures which occur at four-loop order. It is convenient to decompose $\delta Z_{2}^{(4)}$ as

$$
\begin{aligned}
\delta Z_{2}^{(4)}= & C_{F}^{4} \delta Z_{2}^{F F F F}+C_{F}^{3} C_{A} \delta Z_{2}^{F F F A}+C_{F}^{2} C_{A}^{2} \delta Z_{2}^{F F A A}+C_{F} C_{A}^{3} \delta Z_{2}^{F A A A} \\
& +\frac{d_{F}^{a b c d} d_{A}^{a b c d}}{N_{c}} \delta Z_{2}^{d_{F A}}+n_{l} \frac{d_{F}^{a b c d} d_{F}^{a b c d}}{N_{c}} \delta Z_{2}^{d_{F F} L}+n_{h} \frac{d_{F}^{a b c d} d_{F}^{a b c d}}{N_{c}} \delta Z_{2}^{d_{F F} H} \\
& +C_{F}^{3} T n_{l} \delta Z_{2}^{F F F L}+C_{F}^{2} C_{A} T n_{l} \delta Z_{2}^{F F A L}+C_{F} C_{A}^{2} T n_{l} \delta Z_{2}^{F A A L} \\
& +C_{F}^{2} T^{2} n_{l}^{2} \delta Z_{2}^{F F L L}+C_{F} C_{A} T^{2} n_{l}^{2} \delta Z_{2}^{F A L L}+C_{F} T^{3} n_{l}^{3} \delta Z_{2}^{F L L L} \\
& +C_{F}^{3} T n_{h} \delta Z_{2}^{F F F H}+C_{F}^{2} C_{A} T n_{h} \delta Z_{2}^{F F A H}+C_{F} C_{A}^{2} T n_{h} \delta Z_{2}^{F A A H} \\
& +C_{F}^{2} T^{2} n_{h}^{2} \delta Z_{2}^{F F H H}+C_{F} C_{A} T^{2} n_{h}^{2} \delta Z_{2}^{F A H H}+C_{F} T^{3} n_{h}^{3} \delta Z_{2}^{F H H H} \\
& +C_{F}^{2} T^{2} n_{l} n_{h} \delta Z_{2}^{F F L H}+C_{F} C_{A} T^{2} n_{l} n_{h} \delta Z_{2}^{F A L H}+C_{F} T^{3} n_{l}^{2} n_{h} \delta Z_{2}^{F L L H} \\
& +C_{F} T^{3} n_{l} n_{h}^{2} \delta Z_{2}^{F L H H},
\end{aligned}
$$


where $C_{F}, C_{A}, T, n_{l}$, and $n_{h}$ are defined after Eq. (B3) in Appendix B. The new color factors at four loops are the symmetrized traces of four generators in the fundamental and adjoint representation denoted by $d_{F}^{a b c d}$ and $d_{A}^{a b c d}$, respectively.

In Tables V, VI, VII, and VIII (see Appendix A), we show the numerical results for the coefficients introduced in Eq. (13). The numerical uncertainties have been obtained by adding the uncertainties from each individual master integral in quadrature and multiplying the result by a security factor 10 . This approach is quite conservative; however, we observed that there are rare cases where the uncertainty from numerical integration is underestimated by several standard deviations. A factor 10 covers all cases which we have experienced (see also the discussion in Ref. [2]). All coefficients which have a nonzero numerical uncertainty are truncated in such a way that two digits of the uncertainty are shown; otherwise, we present (at least) five significant digits. Note that the $n_{l}^{3}$ and $n_{l}^{2}$ terms are known analytically [17]. None of the other coefficients is known analytically to us, although for some of them, the uncertainty is very small; see, e.g., $C_{F} n_{h}^{3}$.

Let us start with the discussion of Table V. Most of the coefficients are known with an uncertainty of a few percent or below. An exception is the $C_{F}^{4}$ and $C_{F}^{3} C_{A}$ color factors, where the uncertainty is about $30 \%$. In the case of $n_{h}\left(d_{F}^{a b c d}\right)^{2}$, the uncertainty is larger than the central value, and we are not able to decide whether the corresponding coefficient is zero or numerically small. For some color structures, our precision is below a per mille level, in particular for the most non-Abelian color factor $C_{F} C_{A}^{3}$, which provides the numerically largest contribution.

There are some coefficients in the pole parts where the numerical uncertainty is larger than the central value. In these cases, no definite conclusion can be drawn. Within our (conservative) uncertainty estimate, the results are compatible with zero. Still, in these cases, we cannot exclude a small nonzero result. Note, however, that in most cases the uncertainty is much smaller than the central value. In particular, all color structures except those involving $d_{F}^{a b c d}$ or $d_{A}^{a b c d}$ have a nonzero $1 / \epsilon^{4}$ pole. In fact, we expect that the color structures involving $d_{F}^{a b c d}$ and $d_{A}^{a b c d}$ only have a $1 / \epsilon$ pole, which is consistent with our result.

The coefficients in Table VI representing the linear $\xi$ terms are in general much smaller than for $\xi=0$, and the situation is similar as for the pole terms of Table V: we can conclude that the color structures $C_{F}^{2} C_{A}^{2}, C_{F} C_{A}^{3}, d_{F}^{a b c d} d_{A}^{a b c d}$, $C_{F} C_{A}^{2} n_{l}, C_{F}^{2} C_{A} n_{h}, C_{F} C_{A}^{2} n_{h}, C_{F} C_{A} n_{h}^{2}$, and $C_{F} C_{A} n_{l} n_{h}$ have nonzero coefficients. Within our precision, the coefficient of $C_{F}^{3} C_{A}$ is zero; the central value is of order $10^{-4}$ and furthermore ten times smaller than the uncertainty.
However, a closer look into this contribution shows that nontrivial master integrals are involved, which combine to the numerical result given in Table VI. Since the master integrals are linear independent and since they are beyond "three-loop complexity" (i.e., they are neither products of lower-loop integrals nor contain simple one-loop insertions), we would expect a nonzero coefficient unless there are accidental cancellations. Note that at three-loop order there are two color structures which have $\xi$-dependent coefficients: $C_{F} C_{A}^{2}$ and $C_{F} C_{A} n_{h}$.

In Table VII, which contains the $\xi^{2}$ terms, there are nonzero coefficients for the color structures $C_{F} C_{A}^{3}$, $d_{F}^{a b c d} d_{A}^{a b c d}$, and $C_{F} C_{A}^{2} n_{h}$.

It is interesting to check the cancellations between the bare four-loop expression and the mass counterterm contributions (which are known analytically and can be found in the ancillary file for this paper [25]). For this reason, we show in Table VIII the bare four-loop coefficients. The comparison with the corresponding entries in Table V shows that the coefficients of some of the color structures suffer from large cancellations, which in some cases is even more than 2 orders of magnitude (see, e.g., the $C_{F}^{3} n_{l}$ term). Note that the numerically dominant color structure $C_{F} C_{A}^{3}$ is not affected by mass renormalization.

In Ref. [26], the pole of the color structure $n_{l}\left(d_{F}^{a b c d}\right)^{2}$ has been determined from the requirement that a certain combination of renormalization constants in full QCD and HQET are finite (see also the discussion in Sec. III below). Its analytic expression in our notation reads

$$
\begin{aligned}
\delta Z_{2}^{d_{F F} L} & =-\frac{1}{\epsilon}\left(\frac{1}{8}+\frac{\pi^{2}}{12}-\frac{\zeta_{3}}{8}-\frac{\pi^{2} \zeta_{3}}{12}+\frac{5 \zeta_{5}}{32}\right)+\cdots \\
& \approx \frac{0.0294223}{\epsilon}+\cdots,
\end{aligned}
$$

which has to be compared to our numerical result $(0.011 \pm$ $0.064) / \epsilon+\cdots$ (see Table V). The result in Eq. (14) agrees with our result within the uncertainty. Note, however, that the absolute value of this contribution is quite small, which explains our large relative uncertainty.

It is interesting to insert the numerical values of the color factors and evaluate $\delta Z_{2}^{(4)}$ for $N_{c}=3$. To obtain the corresponding expression, we choose $N_{c}=3$ after inserting the master integrals but before combining the uncertainties from the various $\epsilon$ expansion coefficients of the color factors. The results for the various powers of $n_{l}$ are given in Table I. Note that for $\xi=0$ (top) all uncertainties are of order $10^{-4}$. Furthermore, for all powers of $n_{l}$, we observe nonzero coefficients in the poles up to fourth order. For completeness, we present in Table I also results for the $\xi^{1}$ and $\xi^{2}$ terms. For the linear $\xi$ coefficients, we observe nonzero entries only for the $n_{l}^{0}$ and the linear- $n_{l}$ term. The coefficients of $\xi^{2}$ are only nonzero for the $n_{l}^{0}$ contribution. 
TABLE I. Results for the coefficients of $\delta Z_{2}^{(4)}$ after choosing $N_{c}=3$. The $\xi=0, \xi^{1}$, and $\xi^{2}$ contributions are shown in the top, middle, and bottom tables. A security factor 10 has been applied to the uncertainties.

\begin{tabular}{lccccc}
\hline \hline$\xi=0$ & $1 / \epsilon^{4}$ & $1 / \epsilon^{3}$ & $1 / \epsilon^{2}$ & $1 / \epsilon$ & $\epsilon^{0}$ \\
\hline$n_{l}^{0}$ & $-1.77242 \pm 0.00040$ & $-27.6674 \pm 0.0041$ & $-317.093 \pm 0.029$ & $-3142.15 \pm 0.33$ & $-28709.9 \pm 3.2$ \\
$n_{l}^{1}$ & $0.460936 \pm 0.000016$ & $6.69143 \pm 0.00023$ & $74.6540 \pm 0.0013$ & $696.6612 \pm 0.0076$ & $6174.290 \pm 0.084$ \\
$n_{l}^{2}$ & -0.039931 & -0.51572 & -5.5055 & -48.777 & -418.93 \\
$n_{l}^{3}$ & 0.00115741 & 0.0125386 & 0.126757 & 1.07105 & 8.9160 \\
\hline \hline$\xi^{1}$ & $1 / \epsilon^{4}$ & $1 / \epsilon^{3}$ & $1 / \epsilon^{2}$ & $1 / \epsilon$ & $\epsilon^{0}$ \\
\hline$n_{l}^{0}$ & $-0.018555 \pm 0.000011$ & $0.034239 \pm 0.000089$ & $-0.05678 \pm 0.00052$ & $5.2230 \pm 0.0028$ & $36.820 \pm 0.017$ \\
$n_{l}^{1}$ & 0.00173611 & -0.0052083 & 0.0224269 & -0.34863 & -1.61105 \\
\hline \hline$\xi^{2}$ & $1 / \epsilon^{4}$ & $1 / \epsilon^{3}$ & $1 / \epsilon^{2}$ & $1 / \epsilon$ & $\epsilon^{0}$ \\
\hline$n_{l}^{0}$ & $0.0000002 \pm 0.0000038$ & $0.001952 \pm 0.000026$ & $-0.03022 \pm 0.00012$ & $-0.18686 \pm 0.00061$ & $-2.9266 \pm 0.0028$ \\
\hline \hline
\end{tabular}

TABLE II. Results for $Z_{2}^{\mathrm{OS}}$ specified to QED.

\begin{tabular}{cccccc}
\hline \hline & $1 / \epsilon^{4}$ & $1 / \epsilon^{3}$ & $1 / \epsilon^{2}$ & $1 / \epsilon$ & $\epsilon^{0}$ \\
\hline$n_{l}^{0}$ & $0.20500 \pm 0.00037$ & $0.5980 \pm 0.0027$ & $-0.895 \pm 0.021$ & $-6.18 \pm 0.17$ & $-17.4 \pm 1.6$ \\
$n_{l}^{1}$ & $0.17058 \pm 0.00011$ & $0.9556 \pm 0.0014$ & $2.9397 \pm 0.0079$ & $10.480 \pm 0.064$ & $25.92 \pm 0.80$ \\
$n_{l}^{2}$ & 0.056424 & 0.46123 & 3.03509 & 18.7456 & 105.069 \\
$n_{l}^{3}$ & 0.0069444 & 0.075231 & 0.76054 & 6.4263 & 53.496 \\
\hline \hline
\end{tabular}

Finally, we discuss the wave function renormalization for QED. It is obtained from the QCD result by adopting the following values for the QCD color factors:

$$
\begin{aligned}
& C_{F} \rightarrow 1, \quad C_{A} \rightarrow 0, \quad T \rightarrow 1, \quad d_{F}^{a b c d} \rightarrow 1, \\
& d_{A}^{a b c d} \rightarrow 0, \quad N_{c} \rightarrow 1 .
\end{aligned}
$$

We furthermore set $n_{h}=1$ but keep the dependence on $n_{l}$. Note that $n_{l}=0$ corresponds to the case of a massive electron and $n_{l}=1$ describes the case of a massive muon and a massless electron. Our results are shown in Table II. For the $n_{l}$-independent part, we have an uncertainty of about $10 \%$, and the $n_{l}^{1}$ term is determined with a $3 \%$ accuracy.

The on-shell wave function renormalization constant in QED has to be independent of $\xi[5,27]$, which is fulfilled in our result as can be seen from the absence of all Abelian coefficients in Tables VI and VII; they are analytically zero. Note that the gauge parameter dependence only cancels after adding the mass counterterm contributions.

\section{CHECKS AND HQET WAVE FUNCTION RENORMALIZATION}

In this section, we describe several checks of our results. In particular, we discuss the relation to the wave function renormalization constant in HQET.

We start with the discussion of the $\overline{\mathrm{MS}}$ wave function renormalization constant $Z_{2}^{\overline{\mathrm{MS}}}$, which has been obtained to five-loop accuracy in Refs. [28,29]. In these papers, also the full $\xi$-dependence at four loops has been computed, which is crucial for our application. By definition, it only contains ultraviolet poles. On the other hand, as discussed in the Introduction, $Z_{2}^{\mathrm{OS}}$ contains both ultraviolet and infrared poles since it has to take care of both types of divergences in processes containing external heavy quarks. The ultraviolet divergences of $Z_{2}^{\mathrm{OS}}$ have to agree with the ones of $Z_{2}^{\overline{\mathrm{MS}}}$, and thus $Z_{2}^{\overline{\mathrm{MS}}} / Z_{2}^{\mathrm{OS}}$ only contains infrared poles. Note that the latter have to agree with the ultraviolet poles of the wave function renormalization constant in HQET, $Z_{2}^{\mathrm{HQET}}$, which can be seen as follows (see also the discussion in Ref. [5]): the off-shell heavy-quark propagator is infrared finite and contains only ultraviolet divergences, which can be renormalized in the $\overline{\mathrm{MS}}$ scheme; i.e., they are taken care of by $Z_{2}^{\overline{\mathrm{MS}}}$. If one applies an asymptotic expansion [30,31] around the onshell limit, one obtains two contributions. The first one corresponds to a naive Taylor expansion of on-shell integrals which have to be evaluated in full QCD. It develops both ultraviolet and infrared divergences, as 
discussed above for the case of $Z_{2}^{\mathrm{OS}}$. The second contribution corresponds to HQET integrals and only has ultraviolet poles which have to cancel the infrared poles of the QCD contribution. Note that the wave functions $Z_{2}^{\mathrm{OS}}$ and $Z_{2}^{\mathrm{HQET}}$ considered in this paper correspond to the leading term in the expansion and thus $Z_{2}^{\mathrm{HQET}} / Z_{2}^{\mathrm{OS}}$ has to be infrared finite. As a consequence, the following combination of renormalization constants,

$$
\frac{Z_{2}^{\overline{\mathrm{MS}}}}{Z_{2}^{\mathrm{OS}}} Z_{2}^{\mathrm{HQET}}
$$

has to be finite (see also the discussion in Ref. [32]). We will use this fact to determine the poles of $Z_{2}^{\mathrm{HQET}}$.

HQET describes the limit of QCD where the mass of the heavy quark goes to infinity. The heavy-quark field is integrated out from the Lagrange density. Thus, it is not a dynamical degree of freedom anymore. As a consequence, HQET contains as parameters the strong coupling constant and gauge parameter defined in the $n_{l}$-flavor theory, $\alpha_{s}^{\left(n_{l}\right)}$ and $\xi^{\left(n_{l}\right)}{ }^{1}$ Furthermore, there are no closed heavy-quark loops; i.e., color factors involving $n_{h}$ are absent. Thus, when constructing (16), we can check that in the ratio $Z_{2}^{\overline{\mathrm{MS}}} / Z_{2}^{\mathrm{OS}}$ all color structures containing $n_{h}$ are finite after using the decoupling relations for $\alpha_{s}$ and $\xi$ [33]. At two- and three-loop order, this check can be performed analytically. At four loops, we observe that $Z_{2}^{\overline{\mathrm{MS}}} / Z_{2}^{\mathrm{OS}}$ is finite within our numerical precision. Note that this concerns the 11 color structures in Eq. (13) which are proportional to $n_{h}, n_{h}^{2}$, or $n_{h}^{3}$. Let us mention that all coefficients are zero within three standard deviations of the original FIESTA uncertainty, which means that in this case a security factor 3 would be sufficient.

The remaining 12 four-loop color structures are present in $Z_{2}^{\mathrm{HQET}}$, and the corresponding pole term can be extracted from Eq. (16). Before presenting the results, we remark that $Z_{2}^{\mathrm{HQET}}$ exponentiates according to $[5,34]$

$$
\begin{aligned}
Z_{2}^{\mathrm{HQET}}= & \exp \left\{x_{1} C_{F}\left(\frac{\alpha_{s}}{\pi}\right)+C_{F}\left[x_{2} C_{A}+x_{3} T n_{l}\right]\left(\frac{\alpha_{s}}{\pi}\right)^{2}+C_{F}\left[x_{4} C_{A}^{2}+x_{5} C_{A} T n_{l}\right.\right. \\
& \left.+x_{6} T^{2} n_{l}^{2}+x_{7} C_{F} T n_{l}\right]\left(\frac{\alpha_{s}}{\pi}\right)^{3}+\left[C _ { F } \left(x_{8} C_{A}^{3}+x_{9} C_{A}^{2} T n_{l}+x_{10} C_{A} T^{2} n_{l}^{2}\right.\right. \\
& \left.+x_{11} T^{3} n_{l}^{3}+x_{12} C_{F}^{2} T n_{l}+x_{13} C_{F} C_{A} T n_{l}+x_{14} C_{F} T^{2} n_{l}^{2}\right) \\
& \left.\left.+x_{15} d_{F}^{a b c d} d_{A}^{a b c d} / N_{c}+x_{16} d_{F}^{a b c d} d_{F}^{a b c d} n_{l} / N_{c}\right]\left(\frac{\alpha_{s}}{\pi}\right)^{4}+\cdots\right\}
\end{aligned}
$$

and thus there are only nine genuinely new color coefficients at four loops $\left(x_{8}, \ldots, x_{16}\right)$ and the remaining three contributions proportional to $C_{F}^{4}, C_{F}^{3} C_{A}$, and $C_{F}^{2} C_{A}^{2}$ can be predicted from lower loop orders. The comparison with the explicit calculation provides a strong check on our calculation. Note that the predictions of the $C_{F}^{4}, C_{F}^{3} C_{A}$, and $C_{F}^{2} C_{A}^{2}$ contributions are available in analytic form.

In our practical calculations, we proceed as follows. In a first step, we use Eq. (16) to obtain a result for $Z_{2}^{\mathrm{HQET}}$ from the requirement that the combination of the three quantities is finite. Afterward, we use this result and compare to the expanded version of Eq. (17) to determine the coefficients $x_{i}$. Finally, we use Eq. (17) to predict the $C_{F}^{4}, C_{F}^{3} C_{A}$, and $C_{F}^{2} C_{A}^{2}$ of $Z_{2}^{\mathrm{HQET}}$.

\footnotetext{
${ }^{1}$ Note that all quantities discussed in Sec. II depend on $n_{f}=n_{l}+n_{h}$ flavors.
}

We refrain from providing explicit results for $Z_{2}^{\mathrm{HQET}}$ but provide our results for $x_{i}$ in the ancillary file to this paper [25]. Furthermore, we present the expressions for the corresponding anomalous dimension, which is given by

$$
\begin{aligned}
\gamma_{\mathrm{HQET}} & =\frac{\mathrm{d} \log Z_{2}^{\mathrm{HQET}}}{\mathrm{d} \log \mu^{2}} \\
& =\sum_{n \geq 1} \gamma_{\mathrm{HQET}}^{(n)}\left(\frac{\alpha_{s}(\mu)}{\pi}\right)^{n} .
\end{aligned}
$$

Since our four-loop expression for $Z_{2}^{\mathrm{HQET}}$ is only known numerically, we have spurious $\epsilon$ poles in $\gamma_{\text {HQET }}$. However, all of them are zero within two standard deviations of the uncertainty provided by FIESTA, which constitutes another useful cross-check for our calculation.

Let us in the following present our results for $\gamma_{\mathrm{HQET}}$. Up to three-loop order, we have 
TABLE III. Results for the different color factors of $\gamma_{\mathrm{HQET}}^{(4)}$. In columns 2 to 4 , the coefficients of different powers of $\xi^{\left(n_{l}\right)}$ are given. In the uncertainties, a security factor 10 has been introduced.

\begin{tabular}{lccc}
\hline \hline & $\left(\xi^{\left(n_{l}\right)}\right)^{0}$ & $\left(\xi^{\left(n_{l}\right)}\right)^{1}$ & $\left(\xi^{\left(n_{l}\right)}\right)^{2}$ \\
\hline FAAA & $-2.03 \pm 0.35$ & $-0.29037 \pm 0.00052$ & $0.07083 \pm 0.00010$ \\
$d_{F A}$ & $1.53 \pm 0.84$ & $0.5083 \pm 0.0098$ & $-0.1031 \pm 0.0024$ \\
$d_{F F} L$ & $0.54 \pm 0.26$ & & \\
FFFL & $0.1894 \pm 0.0030$ & -0.0076630 & -0.0103348 \\
FFAL & $-0.4566 \pm 0.0055$ & 0.25147 & \\
FAAL & $2.576 \pm 0.010$ & -0.0077460 & \\
FFLL & 0.25725 & & \\
FALL & -0.53745 & & \\
FLLL & -0.048262 & & \\
\hline \hline
\end{tabular}

$$
\begin{aligned}
\gamma_{\mathrm{HQET}}^{(1)}= & -\frac{C_{F}}{2}\left(1+\frac{\xi^{\left(n_{l}\right)}}{2}\right), \\
\gamma_{\mathrm{HQET}}^{(2)}= & C_{F} C_{A}\left(-\frac{19}{24}-\frac{5 \xi^{\left(n_{l}\right)}}{32}+\frac{\left(\xi^{\left(n_{l}\right)}\right)^{2}}{64}\right)+\frac{C_{F} T n_{l}}{3}, \\
\gamma_{\mathrm{HQET}}^{(3)}= & C_{F} C_{A}^{2}\left[-\frac{19495}{27648}-\frac{3 \zeta_{3}}{16}-\frac{\pi^{4}}{360}+\xi^{\left(n_{l}\right)}\left(-\frac{379}{2048}-\frac{15 \zeta_{3}}{256}+\frac{\pi^{4}}{1440}\right)+\left(\xi^{\left(n_{l}\right)}\right)^{2}\left(\frac{69}{2048}+\frac{3 \zeta_{3}}{512}\right)-\frac{5\left(\xi^{\left(n_{l}\right)}\right)^{3}}{1024}\right] \\
& +C_{F} C_{A} T n_{l}\left(\frac{1105}{6912}+\frac{3 \zeta_{3}}{4}+\frac{17 \xi^{\left(n_{l}\right)}}{256}\right)+C_{F}^{2} \operatorname{Tn}_{l}\left(\frac{51}{64}-\frac{3 \zeta_{3}}{4}\right)+\frac{5 C_{F} T^{2} n_{l}^{2}}{108},
\end{aligned}
$$

which agree with Refs. [5,34].

The four-loop terms to $\gamma_{\mathrm{HQET}}$ can be found in Table III, where for each color factor the coefficients of the $\left(\xi^{\left(n_{l}\right)}\right)^{k}$ terms are shown together with their uncertainty. As for $Z_{2}^{\mathrm{OS}}$ in Sec. II, we have introduced a security factor 10 . Note that the coefficients of $\left(\xi^{\left(n_{l}\right)}\right)^{k}$ with $k \geq 3$ have not been computed.
We have the worst precision of about $50 \%$ for the color factors $d_{F}^{a b c d} d_{A}^{a b c d}$ and $n_{l} d_{F}^{a b c d} d_{F}^{a b c d}$ followed by $C_{F} C_{A}^{3}$, which is $17 \%$. The relative uncertainty of the remaining $n_{l}$ terms is much smaller. Note that the $n_{l}^{2}$ and $n_{l}^{3}$ terms are known analytically. They are obtained in a straightforward way for the corresponding analytic results for $Z_{2}^{\mathrm{OS}}$ from Ref. [17]. Our results read

$$
\begin{aligned}
& \gamma_{\mathrm{HQET}}^{(4), F F L L}=\frac{3 \zeta_{3}}{4}-\frac{\pi^{4}}{240}-\frac{103}{432}, \\
& \gamma_{\mathrm{HQET}}^{(4), F A L L}=-\frac{35 \zeta_{3}}{48}+\frac{\pi^{4}}{240}-\frac{4157}{62208}+\xi^{\left(n_{l}\right)}\left(-\frac{\zeta_{3}}{48}+\frac{269}{15552}\right), \\
& \gamma_{\mathrm{HQET}}^{(4), F L L L}=\frac{1}{54}-\frac{\zeta_{3}}{18} .
\end{aligned}
$$

The expression for $\gamma_{\mathrm{HOET}}^{(4), F F L L}$ agrees with Refs. [35,36], and $\gamma_{\mathrm{HQET}}^{(4), F L L L}$ can be found in Ref. [37]. $\gamma_{\mathrm{HQET}}^{(4), F A L L}$ is new.

Recently, also for the $n_{l} d_{F}^{a b c d} d_{F}^{a b c d}$ color structure, analytic results have been obtained [26]. The results read

$$
\gamma_{\mathrm{HQET}}^{(4), d_{F F} L}=-\frac{5}{8} \zeta_{5}+\frac{1}{3} \pi^{2} \zeta_{3}+\frac{1}{2} \zeta_{3}-\frac{1}{3} \pi^{2} \approx 0.617689 \ldots
$$

and agrees well with our findings $\gamma_{\mathrm{HQET}}^{(4), d_{F F} L} \approx 0.54 \pm 0.26$. Note that here a security factor 2 would have been sufficient.
There are no contributions from the color structures $C_{F}^{4}$, $C_{F}^{3} C_{A}$, and $C_{F}^{2} C_{A}^{2}$ to $\gamma_{\mathrm{HQET}}^{(4)}$ as is obvious by inspecting Eq. (17): the four-loop $C_{F}^{4}, C_{F}^{3} C_{A}$, and $C_{F}^{2} C_{A}^{2}$ terms are generated by products of lower-order contributions. Since all coefficients $x_{i}$ only contain poles in $\epsilon$, the $1 / \epsilon$ pole of $Z_{2}^{\mathrm{HQET}}$ does not involve $C_{F}^{4}, C_{F}^{3} C_{A}$, and $C_{F}^{2} C_{A}^{2}$.

Let us finally compare the predicted $C_{F}^{4}, C_{F}^{3} C_{A}$, and $C_{F}^{2} C_{A}^{2}$ contributions to $Z_{2}^{\mathrm{HQET}}$ to the ones we obtain by an explicit calculation. Table IV contains coefficients of $\left(\xi^{\left(n_{l}\right)}\right)^{k} \epsilon^{n}$ for $k=0,1$, and 2 and for values of $n=-4$, $-3, \ldots$ up to one unit higher than the order up to which 
TABLE IV. Contributions of the color structures $C_{F}^{4}, C_{F}^{3} C_{A}$, and $C_{F}^{2} C_{A}^{2}$ to $Z_{2}^{\mathrm{HQET}}$. The coefficients of $\left(\xi^{\left(n_{l}\right)}\right)^{0}$, $\left(\xi^{\left(n_{l}\right)}\right)^{1}$, and $\left(\xi^{\left(n_{l}\right)}\right)^{2}$ are given in rows 2 to 4 . For each power of $\epsilon$, the first row corresponds to the numerical evaluation of the analytic result, and the second row corresponds to the numerical result of our explicit calculation of $Z_{2}^{\mathrm{OS}}$. Relative uncertainties below $10^{-5}$ are set to zero. Note that the uncertainties in this paper are not multiplied by a security factor 10 .

\begin{tabular}{|c|c|c|c|}
\hline & $\left(\xi^{\left(n_{l}\right)}\right)^{0}$ & $\left(\xi^{\left(n_{l}\right)}\right)^{1}$ & $\left(\xi^{\left(n_{l}\right)}\right)^{2}$ \\
\hline \multicolumn{4}{|l|}{$C_{F}^{4}$} \\
\hline \multirow[t]{2}{*}{$1 / \epsilon^{4}$} & 0.0026042 & 0.0052083 & 0.0039063 \\
\hline & $0.0025932 \pm 0.000025$ & 0.0052083 & 0.0039063 \\
\hline \multirow[t]{2}{*}{$1 / \epsilon^{3}$} & 0.00000 & 0.00000 & 0.00000 \\
\hline & $0.00013049 \pm 0.00019$ & 0.00000 & 0.00000 \\
\hline \multicolumn{4}{|c|}{. } \\
\hline \multirow[t]{2}{*}{$1 / \epsilon^{4}$} & 0.035156 & 0.044922 & 0.016602 \\
\hline & $0.035190 \pm 0.00005$ & 0.044922 & 0.016602 \\
\hline \multirow{2}{*}{$1 / \epsilon^{3}$} & -0.049479 & -0.059245 & -0.021159 \\
\hline & $-0.049878 \pm 0.00044$ & $-0.059245 \pm 0.00000006$ & -0.021159 \\
\hline \multirow[t]{2}{*}{$1 / \epsilon^{2}$} & 0.00000 & 0.00000 & 0.00000 \\
\hline & $0.0029893 \pm 0.0041$ & $-0.0000002 \pm 0.0000020$ & 0.00000 \\
\hline \multicolumn{4}{|c|}{$-0.00000020 .0000020$} \\
\hline \multirow[t]{2}{*}{$1 / \epsilon^{4}$} & 0.130914 & 0.085558 & 0.0027262 \\
\hline & $0.130887 \pm 0.00004$ & $0.085558 \pm 0.00000002$ & 0.0027262 \\
\hline \multirow[t]{2}{*}{$1 / \epsilon^{3}$} & -0.31170 & -0.191497 & -0.0081380 \\
\hline & $-0.31133 \pm 0.00035$ & $-0.191497 \pm 0.0000002$ & -0.0081380 \\
\hline \multirow[t]{2}{*}{$1 / \epsilon^{2}$} & 0.27852 & 0.162322 & 0.0088241 \\
\hline & $0.27669 \pm 0.0033$ & $0.162323 \pm 0.000002$ & 0.0088241 \\
\hline \multirow[t]{2}{*}{$1 / \epsilon^{1}$} & 0.00000 & 0.00000 & 0.00000 \\
\hline & $0.046 \pm 0.031$ & $-0.000014 \pm 0.000022$ & 0.00000 \\
\hline
\end{tabular}

the corresponding color structure has a nonzero contribution. The last $\epsilon$ order is shown as a check and demonstrates how well we can reproduce the 0 . Note that in this table the displayed uncertainties are not multiplied by a security factor but correspond to the quadratically combined FIESTA uncertainties. In some cases, the relative uncertainty is very small and thus not shown at all. In all cases shown in Table IV, the numerical results agree within 1.5 sigma with the analytic predictions from Eq. (17). Note the color factors $C_{F}^{4}, C_{F}^{3} C_{A}$, and $C_{F}^{2} C_{A}^{2}$ get contributions from the most complicated master integrals, and thus the above comparison provides a strong check on the numerical setup of our calculation.

\section{CONCLUSIONS}

We have computed four-loop QCD corrections to the wave function renormalization constant of heavy quarks, $Z_{2}^{\mathrm{OS}}$. Besides the on-shell quark mass renormalization constant and the leptonic anomalous magnetic moment, which have been considered in Refs. [1,2,7], respectively, this constitutes a third "classical" application of four-loop on-shell integrals. In the present calculation, we could have largely profited from the previous calculations. However, we had to deal with a more involved reduction to master integrals. Furthermore, we observed higher $\epsilon$ poles in the prefactors of some of the master integrals, which forced us to either change the basis or to expand the corresponding master integrals to higher order in $\epsilon$.

$Z_{2}^{\mathrm{OS}}$ is neither gauge parameter independent nor infrared finite, which excludes two important checks used for $Z_{m}^{\mathrm{OS}}$ and the anomalous magnetic moment. However, a number of cross-checks are provided by the relation to the wave function renormalization constant of HQET.

In physical applications, $Z_{2}^{\mathrm{OS}}$ enters, among other quantities, as a building block. Most likely, in the evaluation of the other pieces, numerical methods play an important role as well, and thus various numerical pieces have to be combined to arrive at physical cross sections or decay rates. It might be that numerical cancellations take place, and thus, to date, it is not clear whether the numerical precision reached for $Z_{2}^{\mathrm{OS}}$ (which is of the order of $10^{-4}$ for $N_{c}=3$ ) is sufficient for phenomenological applications. However, the results obtained in this paper serve for sure as important cross-checks for future more precise or even analytic calculations.

In the future, it would, of course, be desirable to obtain analytic results for fundamental quantities like on-shell QCD renormalization constants such as $Z_{2}^{\mathrm{OS}}$, which is considered in this paper, and $Z_{m}^{\mathrm{OS}}$ from Refs. [1,2]. First steps in this direction have been undertaken in Ref. [23] where a semianalytic approach has been used to obtain a high-precision result for the anomalous magnetic moment of the electron. One could imagine extending this analysis to the QCD-like master integrals. 


\section{ACKNOWLEDGMENTS}

The work of A. S. and V. S. is supported by RFBR, Grant No. 17-02-00175A. We thank the High Performance Computing Center Stuttgart (HLRS) for providing computing time used for the numerical computations with FIESTA. The research was carried out using the equipment of the shared research facilities of HPC computing resources at Lomonosov Moscow State University. P. M. was supported in part by the EU Network HIGGSTOOLS Grant No. PITNGA-2012-316704. We thank Andrey Grozin for carefully reading the manuscript and many useful comments.

\section{APPENDIX A: NUMERICAL RESULTS FOR $Z_{2}^{\mathrm{OS}}$}

Tables V, VI, VII, and VIII contain the numerical results for the coefficients of the individual color factors contributing to $Z_{2}^{\mathrm{OS}}$.

TABLE V. Results for the coefficients of $\delta Z_{2}^{(4)}$ as defined in Eq. (13) for $\xi=0$. A security factor 10 has been applied to the uncertainties.

\begin{tabular}{|c|c|c|c|c|c|}
\hline & $1 / \epsilon^{4}$ & $1 / \epsilon^{3}$ & $1 / \epsilon^{2}$ & $1 / \epsilon$ & $\epsilon^{0}$ \\
\hline$F F F F$ & $0.01317 \pm 0.00025$ & $0.0836 \pm 0.0019$ & $-0.084 \pm 0.017$ & $-1.96 \pm 0.16$ & $-4.1 \pm 1.5$ \\
\hline$F F F A$ & $-0.09665 \pm 0.00053$ & $-0.7611 \pm 0.0044$ & $-1.275 \pm 0.041$ & $1.10 \pm 0.38$ & $-9.8 \pm 3.6$ \\
\hline$F F A A$ & $0.21661 \pm 0.00040$ & $2.1150 \pm 0.0035$ & $9.698 \pm 0.033$ & $57.52 \pm 0.31$ & $324.5 \pm 2.9$ \\
\hline$F A A A$ & $-0.14442 \pm 0.00011$ & $-1.76642 \pm 0.00096$ & $-14.4491 \pm 0.0092$ & $-123.354 \pm 0.086$ & $-1007.40 \pm 0.82$ \\
\hline$d_{F A}$ & $-0.00002 \pm 0.00029$ & $0.0006 \pm 0.0033$ & $-0.002 \pm 0.024$ & $0.40 \pm 0.21$ & $9.4 \pm 2.1$ \\
\hline$d_{F F} L$ & $0.00001 \pm 0.00011$ & $-0.0001 \pm 0.0014$ & $0.0000 \pm 0.0079$ & $0.011 \pm 0.064$ & $-2.18 \pm 0.80$ \\
\hline$d_{F F} H$ & $-0.00001 \pm 0.00023$ & $0.0001 \pm 0.0015$ & $-0.001 \pm 0.011$ & $-0.120 \pm 0.076$ & $0.10 \pm 0.50$ \\
\hline$F F F L$ & $0.0351561 \pm 0.0000013$ & $0.2499987 \pm 0.0000092$ & $0.496651 \pm 0.000077$ & $0.39174 \pm 0.00074$ & $1.3920 \pm 0.0067$ \\
\hline$F F A L$ & $-0.1575519 \pm 0.0000033$ & $-1.457029 \pm 0.000022$ & $-7.60181 \pm 0.00016$ & $-46.0162 \pm 0.0014$ & $-236.417 \pm 0.012$ \\
\hline$F A A L$ & $0.1575515 \pm 0.0000052$ & $1.889980 \pm 0.000070$ & $17.10515 \pm 0.00039$ & $145.3220 \pm 0.0026$ & $1190.195 \pm 0.031$ \\
\hline FFLL & 0.0286458 & 0.244792 & 1.37840 & 8.3824 & 40.329 \\
\hline$F A L L$ & -0.057292 & -0.66059 & -6.3943 & -54.229 & -447.65 \\
\hline$F L L L$ & 0.0069444 & 0.075231 & 0.76054 & 6.4263 & 53.496 \\
\hline$F F F H$ & $0.070313 \pm 0.000023$ & $0.255860 \pm 0.000093$ & $-0.65497 \pm 0.00055$ & $-3.8002 \pm 0.0036$ & $-5.953 \pm 0.019$ \\
\hline$F F A H$ & $-0.26173 \pm 0.00010$ & $-1.58102 \pm 0.00044$ & $-3.2136 \pm 0.0021$ & $-11.729 \pm 0.013$ & $-26.860 \pm 0.083$ \\
\hline$F A A H$ & $0.215336 \pm 0.000061$ & $1.95402 \pm 0.00027$ & $11.5396 \pm 0.0014$ & $70.3186 \pm 0.0091$ & $424.301 \pm 0.056$ \\
\hline FFHH & $0.0937498 \pm 0.0000014$ & $0.2109378 \pm 0.0000059$ & $-0.329095 \pm 0.000035$ & $-0.57438 \pm 0.00013$ & $-7.99681 \pm 0.00079$ \\
\hline$F A H H$ & $-0.117186 \pm 0.000011$ & $-0.681863 \pm 0.000054$ & $-2.52735 \pm 0.00029$ & $-10.3208 \pm 0.0012$ & $-40.2646 \pm 0.0062$ \\
\hline $\mathrm{FHHH}$ & 0.0277778 & 0.047454 & 0.173582 & 0.276902 & 0.61212 \\
\hline$F F L H$ & 0.093750 & 0.50781 & $1.3923245 \pm 0.0000012$ & $5.834231 \pm 0.000010$ & $8.990228 \pm 0.000074$ \\
\hline$F A L H$ & $-0.1545138 \pm 0.0000011$ & $-1.3179979 \pm 0.0000063$ & $-9.088033 \pm 0.000034$ & $-56.32679 \pm 0.00020$ & $-344.7315 \pm 0.0015$ \\
\hline$F L L H$ & 0.0277778 & 0.216435 & 1.65669 & 10.3632 & 64.740 \\
\hline FLHH & 0.041667 & 0.197917 & 1.05074 & 4.2433 & 17.7160 \\
\hline
\end{tabular}

TABLE VI. Same as in Table V but the coefficients of the linear $\xi$ terms.

\begin{tabular}{lccccc}
\hline \hline & $1 / \epsilon^{4}$ & $1 / \epsilon^{3}$ & $1 / \epsilon^{2}$ & $1 / \epsilon$ & $\epsilon^{0}$ \\
\hline$F F F A$ & 0 & 0 & $-0.000000 \pm 0.000020$ & $0.00001 \pm 0.00020$ & $-0.0001 \pm 0.0011$ \\
$F F A A$ & 0 & $-0.0000001 \pm 0.0000016$ & $-0.005369 \pm 0.000022$ & $-0.03679 \pm 0.00022$ & $-0.3166 \pm 0.0012$ \\
$F A A A$ & 0 & $-0.0000001 \pm 0.0000039$ & $0.013200 \pm 0.000023$ & $0.11976 \pm 0.00013$ & $1.42164 \pm 0.00076$ \\
$d_{F A}$ & $-0.0000003 \pm 0.0000100$ & $0.000005 \pm 0.000088$ & $-0.00000 \pm 0.00051$ & $0.1135 \pm 0.0025$ & $0.147 \pm 0.013$ \\
$F A A L$ & 0 & 0 & -0.0035799 & -0.033281 & -0.40121 \\
$F F A H$ & 0.0039062 & -0.0094401 & $0.0069760 \pm 0.0000032$ & $0.037345 \pm 0.000035$ & $-0.76089 \pm 0.00024$ \\
$F A A H$ & -0.0052626 & 0.0112034 & $-0.0854353 \pm 0.0000018$ & $0.216644 \pm 0.000018$ & $-1.40360 \pm 0.00013$ \\
$F A H H$ & 0.00260417 & -0.0078125 & 0.047919 & -0.182917 & 0.78980 \\
$F A L H$ & 0.00173611 & -0.0052083 & 0.043906 & -0.148948 & 0.79619 \\
\hline \hline
\end{tabular}

TABLE VII. Same as in Table V but the coefficients of $\xi^{2}$.

\begin{tabular}{lccccc}
\hline \hline & $1 / \epsilon^{4}$ & $1 / \epsilon^{3}$ & $1 / \epsilon^{2}$ & $1 / \epsilon$ & $\epsilon^{0}$ \\
\hline$F A A A$ & 0 & $0.0000000 \pm 0.0000011$ & $-0.0006711 \pm 0.0000052$ & $-0.005817 \pm 0.000025$ & $-0.07062 \pm 0.00012$ \\
$d_{F A}$ & $0.0000002 \pm 0.0000038$ & $-0.000001 \pm 0.000026$ & $-0.00000 \pm 0.00012$ & $-0.01250 \pm 0.00061$ & $-0.0748 \pm 0.0028$ \\
$F A A H$ & 0 & 0.00032552 & -0.00100945 & 0.0089715 & -0.032896 \\
\hline \hline
\end{tabular}




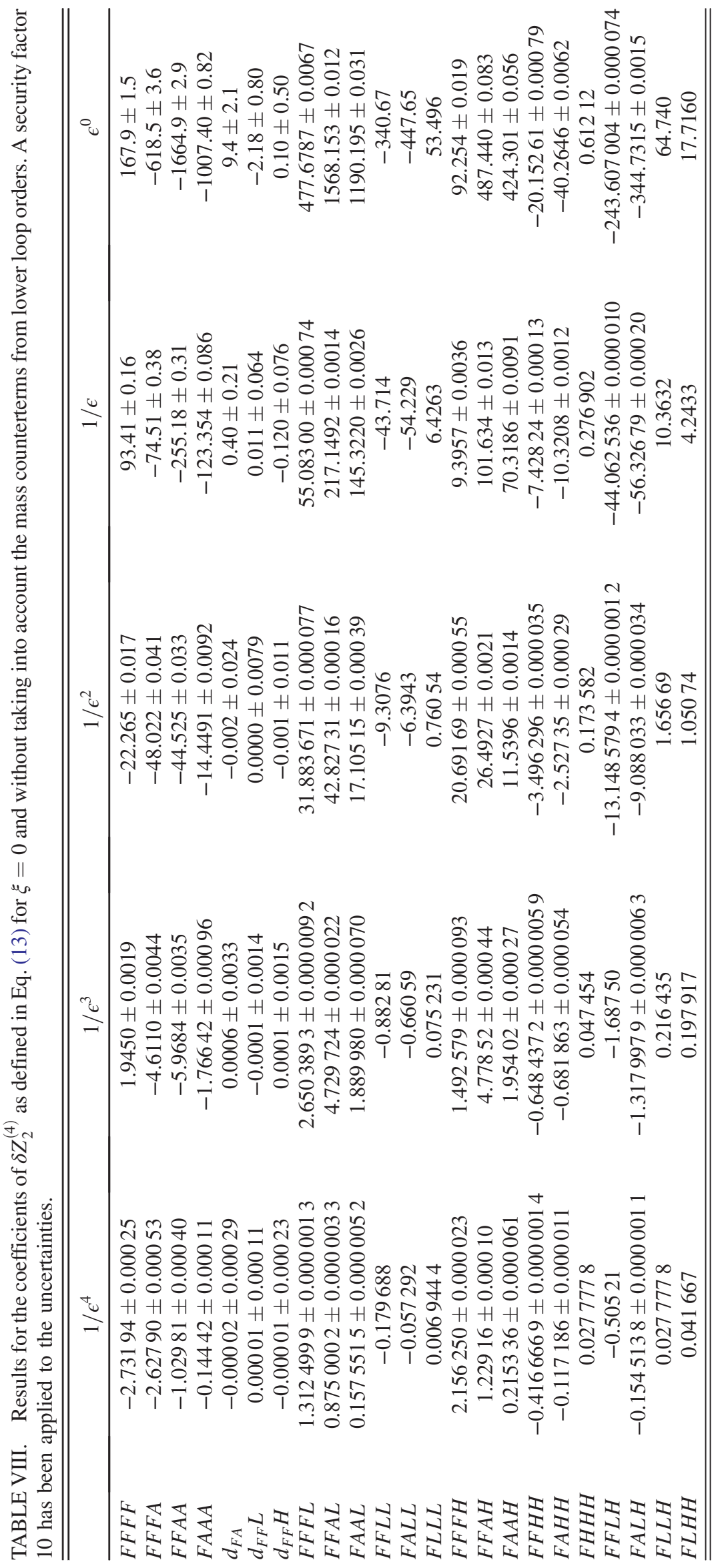




\section{APPENDIX B: $Z_{2}^{\text {OS }}$ TO THREE LOOPS}

In this Appendix, we provide results for the coefficients of $Z_{2}^{\mathrm{OS}}$ as defined in Eq. (9) up to three loops including higherorder terms in $\epsilon$; the $n$-loop expression contains terms up to order $\epsilon^{4-n}$. Note that in Eq. (9) the quark mass $M$ is renormalized on shell but $\alpha_{s}$ is bare. Our results read

$$
\begin{aligned}
& \delta Z_{2}^{(1)}=\left(\frac{\zeta_{3}}{3}-\frac{3 \pi^{4}}{640}-\frac{\pi^{2}}{6}-8\right) \epsilon^{3} C_{F}+\left(\frac{\zeta_{3}}{4}-\frac{\pi^{2}}{12}-4\right) \epsilon^{2} C_{F}+\left(-2-\frac{\pi^{2}}{16}\right) \epsilon C_{F}-\frac{3 C_{F}}{4 \epsilon}-C_{F}, \\
& \delta Z_{2}^{(2)}=\epsilon\left(C_{A} C_{F}\left(12 a_{4}+\frac{199 \zeta_{3}}{24}-\frac{7 \pi^{4}}{40}+\frac{227 \pi^{2}}{384}-\frac{4241}{256}+\frac{\log ^{4}(2)}{2}+\pi^{2} \log ^{2}(2)-\frac{23}{8} \pi^{2} \log (2)\right)\right. \\
& +C_{F}^{2}\left(-24 a_{4}-\frac{297 \zeta_{3}}{16}+\frac{7 \pi^{4}}{20}-\frac{339 \pi^{2}}{128}+\frac{211}{256}-\log ^{4}(2)-2 \pi^{2} \log ^{2}(2)+\frac{23}{4} \pi^{2} \log (2)\right) \\
& \left.+C_{F}\left(-\frac{43 \zeta_{3}}{6}-\frac{437 \pi^{2}}{288}+\frac{20275}{1728}+2 \pi^{2} \log (2)\right) T_{F} n_{h}+\left(\frac{11 \zeta_{3}}{12}+\frac{15 \pi^{2}}{32}+\frac{369}{64}\right) C_{F} T_{F} n_{l}\right) \\
& +\epsilon^{2}\left(C _ { A } C _ { F } \left(69 a_{4}+72 a_{5}-\frac{11 \pi^{2} \zeta_{3}}{8}+\frac{2561 \zeta_{3}}{96}-\frac{609 \zeta_{5}}{8}-\frac{7229 \pi^{4}}{11520}\right.\right. \\
& \left.+\frac{2005 \pi^{2}}{768}-\frac{30163}{512}-\frac{3 \log ^{5}(2)}{5}+\frac{23 \log ^{4}(2)}{8}-2 \pi^{2} \log ^{3}(2)+\frac{23}{4} \pi^{2} \log ^{2}(2)+\frac{13}{30} \pi^{4} \log (2)-\frac{41}{4} \pi^{2} \log (2)\right) \\
& +C_{F}^{2}\left(-138 a_{4}-144 a_{5}+\frac{11 \pi^{2} \zeta_{3}}{4}-\frac{2069 \zeta_{3}}{32}+\frac{609 \zeta_{5}}{4}+\frac{3901 \pi^{4}}{3840}\right. \\
& \left.-\frac{8851 \pi^{2}}{768}+\frac{4889}{512}+\frac{6 \log ^{5}(2)}{5}-\frac{23 \log ^{4}(2)}{4}+4 \pi^{2} \log ^{3}(2)-\frac{23}{2} \pi^{2} \log ^{2}(2)-\frac{13}{15} \pi^{4} \log (2)+\frac{41}{2} \pi^{2} \log (2)\right) \\
& +C_{F} T_{F} n_{h}\left(-48 a_{4}-\frac{2413 \zeta_{3}}{72}+\frac{47 \pi^{4}}{160}-\frac{8509 \pi^{2}}{1728}+\frac{450395}{10368}-2 \log ^{4}(2)-4 \pi^{2} \log ^{2}(2)+\frac{19}{2} \pi^{2} \log (2)\right) \\
& \left.+\left(\frac{33 \zeta_{3}}{8}+\frac{101 \pi^{4}}{960}+\frac{295 \pi^{2}}{192}+\frac{2259}{128}\right) C_{F} T_{F} n_{l}\right) \\
& +C_{A} C_{F}\left(\frac{3 \zeta_{3}}{4}+\frac{49 \pi^{2}}{192}-\frac{803}{128}-\frac{1}{2} \pi^{2} \log (2)\right) \\
& +C_{F}^{2}\left(-\frac{3 \zeta_{3}}{2}-\frac{49 \pi^{2}}{64}+\frac{433}{128}+\pi^{2} \log (2)\right)+\left(\frac{1139}{288}-\frac{7 \pi^{2}}{24}\right) C_{F} T_{F} n_{h} \\
& +\left(\frac{59}{32}+\frac{5 \pi^{2}}{48}\right) C_{F} T_{F} n_{l} \\
& +\frac{-\frac{11 C_{A} C_{F}}{32}+\frac{1}{4} C_{F} T_{F} n_{h}+\frac{1}{8} C_{F} T_{F} n_{l}+\frac{9 C_{F}^{2}}{32}}{\epsilon^{2}} \\
& +\frac{-\frac{101 C_{A} C_{F}}{64}+\frac{19}{48} C_{F} T_{F} n_{h}+\frac{9}{16} C_{F} T_{F} n_{l}+\frac{51 C_{F}^{2}}{64}}{\epsilon} \\
& \delta Z_{2}^{(3)}=\left(-10 a_{4}+\frac{\pi^{2} \zeta_{3}}{8}-\frac{739 \zeta_{3}}{128}-\frac{5 \zeta_{5}}{16}-\frac{5 \log ^{4}(2)}{12}+3 \pi^{2} \log ^{2}(2)+\frac{685}{48} \pi^{2} \log (2)-\frac{41 \pi^{4}}{120}-\frac{58321 \pi^{2}}{9216}-\frac{10823}{3072}\right) C_{F}^{3} \\
& +C_{A}\left(-\frac{319 a_{4}}{6}-\frac{45 \pi^{2} \zeta_{3}}{16}-\frac{19981 \zeta_{3}}{384}+\frac{145 \zeta_{5}}{16}-\frac{319 \log ^{4}(2)}{144}-\frac{499}{72} \pi^{2} \log ^{2}(2)\right. \\
& \left.+\frac{2281}{288} \pi^{2} \log (2)+\frac{20053 \pi^{4}}{17280}-\frac{15053 \pi^{2}}{9216}+\frac{150871}{9216}\right) C_{F}^{2}
\end{aligned}
$$




$$
\begin{aligned}
& +T_{F} n_{l}\left(\frac{64 a_{4}}{3}+\frac{1661 \zeta_{3}}{96}+\frac{8 \log ^{4}(2)}{9}+\frac{16}{9} \pi^{2} \log ^{2}(2)-\frac{58}{9} \pi^{2} \log (2)-\frac{733 \pi^{4}}{2160}+\frac{6931 \pi^{2}}{2304}-\frac{3773}{2304}\right) C_{F}^{2} \\
& +T_{F} n_{h}\left(28 a_{4}+\frac{5327 \zeta_{3}}{288}+\frac{7 \log ^{4}(2)}{6}+\frac{5}{6} \pi^{2} \log ^{2}(2)-\frac{31}{9} \pi^{2} \log (2)-\frac{137 \pi^{4}}{720}+\frac{25223 \pi^{2}}{20736}-\frac{78967}{6912}\right) C_{F}^{2} \\
& +T_{F}^{2} n_{l}^{2}\left(-\frac{37 \zeta_{3}}{36}-\frac{23 \pi^{2}}{48}-\frac{4025}{972}\right) C_{F}+T_{F}^{2} n_{h} n_{l}\left(\frac{49 \zeta_{3}}{12}-\frac{4}{3} \pi^{2} \log (2)+\frac{77 \pi^{2}}{72}-\frac{1168}{81}\right) C_{F} \\
& +T_{F}^{2} n_{h}^{2}\left(\frac{85 \zeta_{3}}{12}-\frac{4}{3} \pi^{2} \log (2)+\frac{767 \pi^{2}}{720}-\frac{6887}{648}\right) C_{F}+C_{A} T_{F} n_{h}\left(-16 a_{4}+\xi\left(-\frac{7 \zeta_{3}}{192}+\frac{\pi^{2}}{256}+\frac{407}{1728}\right)\right. \\
& \left.+\frac{11 \pi^{2} \zeta_{3}}{48}-\frac{3359 \zeta_{3}}{144}-\frac{15 \zeta_{5}}{16}-\frac{2 \log ^{4}(2)}{3}-\frac{1}{3} \pi^{2} \log ^{2}(2)+\frac{521}{36} \pi^{2} \log (2)+\frac{5 \pi^{4}}{72}-\frac{105359 \pi^{2}}{10368}+\frac{32257}{648}\right) C_{F} \\
& +C_{A} T_{F} n_{l}\left(-\frac{32 a_{4}}{3}-\frac{301 \zeta_{3}}{72}-\frac{4 \log ^{4}(2)}{9}-\frac{8}{9} \pi^{2} \log ^{2}(2)+\frac{29}{9} \pi^{2} \log (2)+\frac{29 \pi^{4}}{216}+\frac{2413 \pi^{2}}{3456}+\frac{416405}{15552}\right) C_{F} \\
& +C_{A}^{2}\left(\frac{349 a_{4}}{12}+\frac{127 \pi^{2} \zeta_{3}}{72}+\frac{3623 \zeta_{3}}{144}+\xi\left(\frac{\pi^{2} \zeta_{3}}{144}-\frac{13 \zeta_{3}}{256}+\frac{7 \zeta_{5}}{384}+\frac{17 \pi^{4}}{27648}-\frac{\pi^{2}}{256}-\frac{13}{768}\right)\right. \\
& \left.-\frac{37 \zeta_{5}}{6}+\frac{349 \log ^{4}(2)}{288}+\frac{391}{144} \pi^{2} \log ^{2}(2)-\frac{271}{36} \pi^{2} \log (2)-\frac{10811 \pi^{4}}{23040}-\frac{107 \pi^{2}}{864}-\frac{2551697}{62208}\right) C_{F} \\
& +\frac{1}{\epsilon^{3}}\left[-\frac{9 C_{F}^{3}}{128}+\frac{33}{128} C_{A} C_{F}^{2}-\frac{3}{16} T_{F} n_{h} C_{F}^{2}-\frac{3}{32} T_{F} n_{l} C_{F}^{2}-\frac{121}{576} C_{A}^{2} C_{F}\right. \\
& \left.-\frac{1}{12} T_{F}^{2} n_{h}^{2} C_{F}-\frac{1}{36} T_{F}^{2} n_{l}^{2} C_{F}+\frac{11}{72} C_{A} T_{F} n_{l} C_{F}-\frac{1}{12} T_{F}^{2} n_{h} n_{l} C_{F}+C_{A} T_{F} n_{h}\left(\frac{15}{64}-\frac{\xi}{192}\right) C_{F}\right] \\
& +\frac{1}{\epsilon^{2}}\left[-\frac{81 C_{F}^{3}}{256}+\frac{1217}{768} C_{A} C_{F}^{2}-\frac{91}{192} T_{F} n_{h} C_{F}^{2}-\frac{103}{192} T_{F} n_{l} C_{F}^{2}-\frac{1501}{864} C_{A}^{2} C_{F}\right. \\
& \left.-\frac{5}{36} T_{F}^{2} n_{h}^{2} C_{F}-\frac{23}{108} T_{F}^{2} n_{l}^{2} C_{F}+\frac{1069}{864} C_{A} T_{F} n_{l} C_{F}-\frac{7}{18} T_{F}^{2} n_{h} n_{l} C_{F}+C_{A} T_{F} n_{h}\left(\frac{\xi}{64}+\frac{353}{288}\right) C_{F}\right] \\
& +\frac{1}{\epsilon}\left[\left(\frac{9 \zeta_{3}}{8}-\frac{3}{4} \pi^{2} \log (2)+\frac{303 \pi^{2}}{512}-\frac{1039}{512}\right) C_{F}^{3}+T_{F} n_{l}\left(\frac{3 \zeta_{3}}{4}-\frac{2}{3} \pi^{2} \log (2)+\frac{175 \pi^{2}}{384}-\frac{351}{128}\right) C_{F}^{2}\right. \\
& +T_{F} n_{h}\left(\zeta_{3}-\frac{2}{3} \pi^{2} \log (2)+\frac{143 \pi^{2}}{192}-\frac{1525}{384}\right) C_{F}^{2}+C_{A}\left(-\frac{27 \zeta_{3}}{8}+\frac{53}{24} \pi^{2} \log (2)-\frac{2549 \pi^{2}}{1536}+\frac{14887}{1536}\right) C_{F}^{2} \\
& +C_{A}^{2}\left(\xi\left(-\frac{3 \zeta_{3}}{256}+\frac{\pi^{4}}{4320}-\frac{1}{768}\right)+\frac{173 \zeta_{3}}{128}-\frac{11}{12} \pi^{2} \log (2)-\frac{\pi^{4}}{1080}+\frac{1199 \pi^{2}}{2304}-\frac{55945}{5184}\right) C_{F} \\
& +C_{A} T_{F} n_{h}\left(\left(-\frac{35}{576}-\frac{\pi^{2}}{768}\right) \xi-\frac{\zeta_{3}}{2}+\frac{1}{3} \pi^{2} \log (2)-\frac{1753 \pi^{2}}{2304}+\frac{503}{48}\right) C_{F} \\
& +C_{A} T_{F} n_{l}\left(-\frac{\zeta_{3}}{4}+\frac{1}{3} \pi^{2} \log (2)-\frac{5 \pi^{2}}{288}+\frac{550}{81}\right) C_{F}+T_{F}^{2} n_{h}^{2}\left(-\frac{131}{54}+\frac{29 \pi^{2}}{144}\right) C_{F} \\
& \left.+T_{F}^{2} n_{h} n_{l}\left(-\frac{31}{9}+\frac{7 \pi^{2}}{48}\right) C_{F}+T_{F}^{2} n_{l}^{2}\left(-\frac{325}{324}-\frac{\pi^{2}}{16}\right) C_{F}\right] \\
& +\epsilon\left(\left(\frac{29 \zeta_{3}^{2}}{32}+14 \pi^{2} \log (2) \zeta_{3}+\frac{5267 \pi^{2} \zeta_{3}}{288}-\frac{69511 \zeta_{3}}{256}-\frac{4267 a_{4}}{6}-\frac{116 a_{5}}{3}\right.\right. \\
& -\frac{1403 \zeta_{5}}{16}+\frac{29 \log ^{5}(2)}{90}+\frac{2}{3} \pi^{2} \log ^{4}(2)-\frac{4267 \log ^{4}(2)}{144}-\frac{196}{27} \pi^{2} \log ^{3}(2) \\
& -\frac{2}{3} \pi^{4} \log ^{2}(2)-\frac{3997}{72} \pi^{2} \log ^{2}(2)-\frac{2351 \pi^{4} \log (2)}{2160}+\frac{1345}{8} \pi^{2} \log (2)-\frac{899 \pi^{6}}{5670}
\end{aligned}
$$




$$
\begin{aligned}
& \left.+\frac{450893 \pi^{4}}{552960}+16 a_{4} \pi^{2}-\frac{56455 \pi^{2}}{2048}-\frac{108677}{6144}\right) C_{F}^{3} \\
& +T_{F} n_{l}\left(\frac{1856 a_{4}}{9}+\frac{512 a_{5}}{3}-\frac{69 \pi^{2} \zeta_{3}}{16}+\frac{57581 \zeta_{3}}{576}-\frac{2245 \zeta_{5}}{12}-\frac{64 \log ^{5}(2)}{45}\right. \\
& +\frac{232 \log ^{4}(2)}{27}-\frac{128}{27} \pi^{2} \log ^{3}(2)+\frac{464}{27} \pi^{2} \log ^{2}(2)+\frac{139}{270} \pi^{4} \log (2)-\frac{908}{27} \pi^{2} \log (2) \\
& \left.-\frac{610451 \pi^{4}}{414720}+\frac{251107 \pi^{2}}{13824}-\frac{36677}{13824}\right) C_{F}^{2} \\
& +T_{F} n_{h}\left(\frac{539 a_{4}}{6}+168 a_{5}-\frac{287 \pi^{2} \zeta_{3}}{24}+\frac{1087 \zeta_{3}}{96}-\frac{899 \zeta_{5}}{8}-\frac{7 \log ^{5}(2)}{5}\right. \\
& +\frac{539 \log ^{4}(2)}{144}-\frac{5}{3} \pi^{2} \log ^{3}(2)-\frac{683}{144} \pi^{2} \log ^{2}(2)+\frac{247}{180} \pi^{4} \log (2)+\frac{673}{24} \pi^{2} \log (2) \\
& \left.-\frac{32857 \pi^{4}}{69120}-\frac{341735 \pi^{2}}{41472}-\frac{143029}{13824}\right) C_{F}^{2} \\
& +C_{A}\left(-\frac{143 \zeta_{3}^{2}}{4}+\frac{21}{4} \pi^{2} \log (2) \zeta_{3}-\frac{5777 \pi^{2} \zeta_{3}}{384}-\frac{188083 \zeta_{3}}{2304}-\frac{3787 a_{4}}{36}\right. \\
& -\frac{1441 a_{5}}{3}+\frac{254581 \zeta_{5}}{384}+\frac{1441 \log ^{5}(2)}{360}+\frac{1}{4} \pi^{2} \log ^{4}(2)-\frac{3787 \log ^{4}(2)}{864} \\
& +\frac{2089}{108} \pi^{2} \log ^{3}(2)-\frac{1}{4} \pi^{4} \log ^{2}(2)-\frac{25441}{432} \pi^{2} \log ^{2}(2)-\frac{559}{540} \pi^{4} \log (2) \\
& \left.-\frac{5623}{216} \pi^{2} \log (2)-\frac{27331 \pi^{6}}{181440}+\frac{11810161 \pi^{4}}{1658880}+6 a_{4} \pi^{2}-\frac{1265393 \pi^{2}}{55296}+\frac{4824655}{55296}\right) C_{F}^{2} \\
& +T_{F}^{2} n_{l}^{2}\left(-\frac{851 \zeta_{3}}{108}-\frac{535 \pi^{4}}{3456}-\frac{325 \pi^{2}}{144}-\frac{23789}{1458}\right) C_{F} \\
& +T_{F}^{2} n_{h} n_{l}\left(\frac{128 a_{4}}{3}+\frac{595 \zeta_{3}}{18}+\frac{16 \log ^{4}(2)}{9}+\frac{32}{9} \pi^{2} \log ^{2}(2)-\frac{92}{9} \pi^{2} \log (2)-\frac{341 \pi^{4}}{3456}+\frac{145 \pi^{2}}{36}-\frac{17740}{243}\right) C_{F} \\
& +T_{F}^{2} n_{h}^{2}\left(\frac{152 a_{4}}{3}+\frac{25723 \zeta_{3}}{720}+\frac{19 \log ^{4}(2)}{9}+\frac{17}{9} \pi^{2} \log ^{2}(2)-\frac{33}{5} \pi^{2} \log (2)-\frac{391 \pi^{4}}{1152}+\frac{83227 \pi^{2}}{16200}-\frac{1023397}{19440}\right) C_{F} \\
& +C_{A}^{2}\left(\frac{7451 \zeta_{3}^{2}}{384}-\frac{49}{8} \pi^{2} \log (2) \zeta_{3}+\frac{3299 \pi^{2} \zeta_{3}}{4608}+\frac{236171 \zeta_{3}}{3456}+\frac{4147 a_{4}}{18}+\frac{1499 a_{5}}{6}\right. \\
& +\xi\left(\frac{25 \zeta_{3}^{2}}{384}+\frac{77 \pi^{2} \zeta_{3}}{9216}-\frac{63 \zeta_{3}}{256}-\frac{149 \zeta_{5}}{768}+\frac{49 \pi^{6}}{51840}+\frac{383 \pi^{4}}{138240}-\frac{35 \pi^{2}}{1024}-\frac{35}{256}\right) \\
& -\frac{77297 \zeta_{5}}{256}-\frac{1499 \log ^{5}(2)}{720}-\frac{7}{24} \pi^{2} \log ^{4}(2)+\frac{4147 \log ^{4}(2)}{432}-\frac{1697}{216} \pi^{2} \log ^{3}(2) \\
& +\frac{7}{24} \pi^{4} \log ^{2}(2)+\frac{4679}{108} \pi^{2} \log ^{2}(2)+\frac{6823 \pi^{4} \log (2)}{8640}-\frac{25069}{864} \pi^{2} \log (2) \\
& \left.+\frac{45047 \pi^{6}}{362880}-\frac{2973217 \pi^{4}}{829440}-7 a_{4} \pi^{2}+\frac{26425 \pi^{2}}{20736}-\frac{74570603}{373248}\right) C_{F} \\
& +C_{A} T_{F} n_{l}\left(-\frac{928 a_{4}}{9}-\frac{256 a_{5}}{3}+\frac{77 \pi^{2} \zeta_{3}}{48}-\frac{11159 \zeta_{3}}{864}+\frac{2077 \zeta_{5}}{24}+\frac{32 \log ^{5}(2)}{45}-\frac{116 \log ^{4}(2)}{27}+\frac{64}{27} \pi^{2} \log ^{3}(2)\right. \\
& \left.-\frac{232}{27} \pi^{2} \log ^{2}(2)-\frac{139}{540} \pi^{4} \log (2)+\frac{454}{27} \pi^{2} \log (2)+\frac{122131 \pi^{4}}{103680}+\frac{10535 \pi^{2}}{2592}+\frac{10805903}{93312}\right) C_{F}
\end{aligned}
$$




$$
\begin{aligned}
& +C_{A} T_{F} n_{h}\left(\frac{181 \zeta_{3}^{2}}{32}+7 \pi^{2} \log (2) \zeta_{3}+\frac{1823 \pi^{2} \zeta_{3}}{192}-\frac{357881 \zeta_{3}}{1152}-\frac{5855 a_{4}}{12}-96 a_{5}\right. \\
& +\xi\left(\frac{7 \zeta_{3}}{64}+\frac{173 \pi^{4}}{92160}-\frac{35 \pi^{2}}{2304}-\frac{4859}{5184}\right)+\frac{2697 \zeta_{5}}{64}+\frac{4 \log ^{5}(2)}{5}+\frac{1}{3} \pi^{2} \log ^{4}(2) \\
& -\frac{5855 \log ^{4}(2)}{288}+\frac{2}{3} \pi^{2} \log ^{3}(2)-\frac{1}{3} \pi^{4} \log ^{2}(2)-\frac{21349}{288} \pi^{2} \log ^{2}(2)-\frac{149}{180} \pi^{4} \log (2) \\
& \left.\left.+\frac{3973}{36} \pi^{2} \log (2)-\frac{1501 \pi^{6}}{15120}+\frac{173713 \pi^{4}}{55296}+8 a_{4} \pi^{2}-\frac{163981 \pi^{2}}{5184}+\frac{1004165}{3888}\right) C_{F}\right),
\end{aligned}
$$

where $C_{F}=\left(N_{c}^{2}-1\right) /\left(2 N_{c}\right)$ and $C_{A}=N_{c}$ are the eigenvalues of the quadratic Casimir operators of the fundamental and adjoint representation for the $\mathrm{SU}\left(N_{c}\right)$ color group, respectively; $T=1 / 2$ is the index of the fundamental representation; and $n_{l}$ and $n_{h}$ count the number of massless and massive (with mass $M$ ) quarks. It is convenient to keep the variable $n_{h}$ as a parameter, although in our case, we have $n_{h}=1$. Computer-readable expressions of $\delta Z_{2}^{(1)}, \delta Z_{2}^{(2)}$, and $\delta Z_{2}^{(3)}$ can be found in Ref. [25].

[1] P. Marquard, A. V. Smirnov, V. A. Smirnov, and M. Steinhauser, Phys. Rev. Lett. 114, 142002 (2015).

[2] P. Marquard, A. V. Smirnov, V. A. Smirnov, M. Steinhauser, and D. Wellmann, Phys. Rev. D 94, 074025 (2016).

[3] N. Gray, D. J. Broadhurst, W. Grafe, and K. Schilcher, Z. Phys. C 48, 673 (1990).

[4] K. Melnikov and T. v. Ritbergen, Phys. Lett. B 482, 99 (2000).

[5] K. Melnikov and T. van Ritbergen, Nucl. Phys. B591, 515 (2000).

[6] P. Marquard, L. Mihaila, J. H. Piclum, and M. Steinhauser, Nucl. Phys. B773, 1 (2007).

[7] P. Marquard, A. V. Smirnov, V. A. Smirnov, M. Steinhauser, and D. Wellmann, arXiv:1708.07138.

[8] A. V. Smirnov, J. High Energy Phys. 10 (2008) 107.

[9] A. V. Smirnov and V. A. Smirnov, Comput. Phys. Commun. 184, 2820 (2013).

[10] A. V. Smirnov, Comput. Phys. Commun. 189, 182 (2015).

[11] R. N. Lee, arXiv:1212.2685.

[12] R. N. Lee, J. Phys. Conf. Ser. 523, 012059 (2014).

[13] P. Marquard and D. Seidel (unpublished).

[14] A. V. Smirnov and M. N. Tentyukov, Comput. Phys. Commun. 180, 735 (2009).

[15] A. V. Smirnov, V. A. Smirnov, and M. Tentyukov, Comput. Phys. Commun. 182, 790 (2011).

[16] A. V. Smirnov, Comput. Phys. Commun. 185, 2090 (2014).

[17] R. Lee, P. Marquard, A. V. Smirnov, V. A. Smirnov, and M. Steinhauser, J. High Energy Phys. 03 (2013) 162.

[18] V. A. Smirnov, Phys. Lett. B 460, 397 (1999).

[19] J. B. Tausk, Phys. Lett. B 469, 225 (1999).

[20] M. Czakon, Comput. Phys. Commun. 175, 559 (2006).
[21] A. V. Smirnov and V. A. Smirnov, Eur. Phys. J. C 62, 445 (2009).

[22] H. R. P. Ferguson and D. H. Bailey, RNR, Technical Report RNR-91-032; H. R. P. Ferguson, D. H. Bailey, and S. Arno, NASA, Technical Report NAS-96-005.

[23] S. Laporta, Phys. Lett. B 772, 232 (2017).

[24] D. J. Broadhurst, N. Gray, and K. Schilcher, Z. Phys. C 52, 111 (1991).

[25] See Supplemental Material at http://link.aps.org/ supplemental/10.1103/PhysRevD.97.054032 for the complete analytic results of $Z_{2}^{\mathrm{OS}}$ up to three loops and the analytic mass counterterm contribution at four loops.

[26] A. Grozin, J. Henn, and M. Stahlhofen, J. High Energy Phys. 10 (2017) 052.

[27] K. Johnson and B. Zumino, Phys. Rev. Lett. 3, 351 (1959).

[28] T. Luthe, A. Maier, P. Marquard, and Y. Schroder, J. High Energy Phys. 01 (2017) 081.

[29] P. A. Baikov, K. G. Chetyrkin, and J. H. Kühn, J. High Energy Phys. 04 (2017) 119.

[30] M. Beneke and V. A. Smirnov, Nucl. Phys. B522, 321 (1998).

[31] V. A. Smirnov, Springer Tracts Mod. Phys. 250, 1 (2012).

[32] A. G. Grozin, Phys. Lett. B 692, 161 (2010).

[33] K. G. Chetyrkin, B. A. Kniehl, and M. Steinhauser, Nucl. Phys. B510, 61 (1998).

[34] K. G. Chetyrkin and A. G. Grozin, Nucl. Phys. B666, 289 (2003).

[35] A. Grozin, J. M. Henn, G. P. Korchemsky, and P. Marquard, J. High Energy Phys. 01 (2016) 140.

[36] A. Grozin, Proc. Sci., LL2016 (2016) 053.

[37] D. J. Broadhurst and A. G. Grozin, Phys. Rev. D 52, 4082 (1995). 